\title{
A theoretical investigation on the spectrum of the Ar trimer for high rotational excitations
}

\author{
M. Márquez-Mijares, ${ }^{1,2}$ R. Pérez de Tudela, ${ }^{1}$ T. González-Lezana, ${ }^{1, a)}$ O. Roncero, ${ }_{4}^{1}$ \\ S. Miret-Artés, ${ }^{1}$ G. Delgado-Barrio, ${ }^{1}$ P. Villarreal, ${ }^{1}$ I. Baccarelli, ${ }^{3}$ F. A. Gianturco, ${ }^{4}$ and \\ J. Rubayo-Soneira ${ }^{2}$ \\ ${ }^{1}$ Instituto de Física Fundamental (CSIC), Serrano 123, 28006 Madrid, Spain \\ ${ }^{2}$ Instituto Superior de Tecnologías y Ciencias Aplicadas, Ave. Salvador Allende y Luaces Quinta \\ de Los Molinos, Plaza, La Habana 10600, Cuba \\ ${ }^{3}$ Supercomputing Consortium for University and Research, CASPUR, via dei Tizii 6, 00185 Rome, \\ Italy \\ ${ }^{4}$ Department of Chemistry, University of Rome La Sapienza, Piazzale A. Moro 5, 00185 Rome, \\ Italy
}

(Received 19 December 2008; accepted 17 March 2009; published online 15 April 2009)

\begin{abstract}
A detailed study of the rovibrational spectrum of the Ar trimer is performed by means of an exact hyperspherical coordinate (HC) method and a variational approach based on distributed Gaussian functions (DGFs) to describe the interparticle distances. The good agreement observed between the energy levels obtained with both procedures for high values of the total angular momentum ( $J=15$ and 20) reveals the quality of the DGF method to describe the rotation of the title system. Rotational constants for the lowest bound states, obtained as averages for each vibrational state, have been obtained and compared to previous results. A detailed analysis of density probability functions obtained by means of the HC approach for rovibrational states at $J=0$ and 20 shows close similitudes thus supporting the vibration-rotation separation adopted within the DGF scheme for the $\mathrm{Ar}_{3}$ system. (C) 2009 American Institute of Physics. [DOI: 10.1063/1.3115100]
\end{abstract}

\section{INTRODUCTION}

The calculation of the vibration-rotation (VR) spectrum of polyatomic molecules has been the subject of numerous theoretical investigations over several decades. ${ }^{1}$ Succesive improvements in the experimental techniques have enabled the acquisition of very highly resolved and accurate spectra whose complete understanding requires the parallel development of rigorous quantum mechanical treatments. With this goal, different expressions for rovibrational Hamiltonians for polyatomic molecules have been proposed since the late 30 s. $^{2-4}$ A possible way to tackle the calculation of the VR energy levels is to separate the purely vibrational kinetic energy operator from the corresponding operator for the VR part: ${ }^{1}$ one can then employ, in order to solve the full VR problem, a basis set formed with eigenstates of the vibrational Hamiltonian plus standard rotational basis functions. ${ }^{2,3,5-11}$ This was found to be a convenient approach in the case of trimers such as $\mathrm{KCN},{ }^{8} \mathrm{H}_{2} \mathrm{Ne},{ }^{8} \mathrm{H}_{2} \mathrm{O}$, ${ }^{9}$ and $\mathrm{H}_{3}^{+}$. Tennyson and Sutcliffe ${ }^{11}$ studied the highly excited rotational states of the $\mathrm{H}_{2} \mathrm{D}^{+}$system by means of a similar method in which the diagonal part of the VR Hamiltonian was also considered, besides the Hamiltonian for the zero total angular momentum $(J=0)$ problem, to obtain the corresponding vibrational basis set.

In line with previous applications of this type of strategy, we have recently proposed an approximate variational quantum mechanical approach to study rotating van der Waals

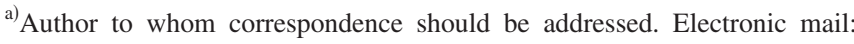
tglezana@imaff.cfmac.csic.es.
}

trimers $^{12}$ based on the use of distributed Gaussian functions ${ }^{13}$ (DGFs) to describe the interparticle distances. A variant of this DGF method was originally developed to study the rotationless spectrum of weakly bound systems as the $\mathrm{He}_{3}$ (Refs. 14-16) and $\mathrm{LiHe}_{2}$ (Refs. 17 and 18) trimers. Since then, the energy levels and geometries of a large series of three-body (3B) systems have been analyzed in detail for the case of $J=0 .{ }^{19,20}$ Based on previous attempts to deal with the case of $J>0,{ }^{20,21}$ a possible scheme to obtain the rovibrational bound states of the Ar trimer was also suggested. ${ }^{12}$ Although the total symmetry is, in principle, not imposed in the DGF calculation, the classification of the energy levels within the corresponding symmetry irreducible representations (IRs) can be done according to the values of the $\Omega$ and $\ell$ quantum numbers for the $z$-component of the total angular momentum in the body-fixed reference frame and the vibrational angular momentum, respectively. The $\ell$ quantum number can be assigned to the $k$ vibrational states by a simple comparison with the spectrum obtained in a hyperspherical coordinate (HC) calculation at $J=0$. The agreement between the predicted rovibrational spectra for values of $J$ up to 6 with energy levels calculated with the HC calculation reveals that such an approach successfully describes the rotation of the $\mathrm{Ar}_{3}$ system.

The Ar trimer has been extensively studied in previous occasions $^{15,22-32}$ and its energy levels and geometries of the bound states for $J=0$ have been analyzed in detail by means of different theoretical approaches. The more rigid and spatially localized nature of this trimer in comparison with systems formed with lighter rare gas atoms such as $\mathrm{Ne}$ or 
$\mathrm{He},{ }^{15,29}$ explains why one of the most commonly investigated issues regards the possible role played by $3 \mathrm{~B}$ terms in the overall potential energy surface (PES) responsible for the internal structure of $\mathrm{Ar}_{3}{ }^{23,25,26,32-34}$ In particular, the inclusion of the Axilrod-Teller (AT) term was found to introduce significant differences in the average geometry of some of the bound states of its vibrational spectrum. ${ }^{32}$ Unfortunately, the absence of a permanent dipole moment and the extremely small transient dipole of this system induced by the deviations from the equilateral structure ${ }^{25,35}$ hinder, however, possible attempts at measuring its far-infrared absorption spectrum to detect these effects. 3B interactions for $\mathrm{Ar}_{3}$ have been also theoretically investigated by means of $a b$ initio calculations. $^{33,36,37}$ In particular, in a recent $\mathrm{HC}$ study a newly developed $a b$ initio 3B PES was employed to calculate the rovibrational spectra of $\mathrm{Ar}_{3}$ for total angular momenta up to $J=6 .^{37}$ In this present work, we have used the approximate DGF method for rotating trimers to obtain the energy levels of the title system for even larger values of the total angular momentum, $J=15$ and 20.

The study of the rovibrational spectrum of the $\mathrm{Ar}_{3}$ system is completed with the calculation of the rotational constants $A, B$, and $C$ for the bound states obtained for $J=0$. The results are compared with values reported in previous calculations on the system and employed in analytical expressions for the rovibrational spectrum of a rigid asymmetric rotor. ${ }^{7} \mathrm{~A}$ similar analysis has been recently performed for the $\mathrm{Ne}$ trimer, ${ }^{38}$ where the $\mathrm{Ne}_{3}$ system was treated as an oblate symmetric top. The spectrum obtained from the rotational constants for each vibrational state and the one predicted by the general DGF approach, where the rotational Hamiltonian matrix contains possible interactions between different vibrational states, are compared.

This paper is structured as follows: In Sec. II the theoretical details of both the HC and DGF calculation are given. In Sec. III we refer to the PES employed here and to some numerical details concerning the calculations. Results are shown in Sec. IV, where the case of $J=0$ and $J>0$ are treated separately. This section ends with the results of the present study regarding the rotational constants of the $\mathrm{Ar}_{3}$ system. The discussion and final conclusions are presented in Secs. V and VI.

\section{THEORY}

\section{A. Hyperspherical coordinate method}

Exact calculations of rovibrational states have been performed using the $\mathrm{HC}$ method of Pack and Parker, ${ }^{39}$ which is closely related to that described by Smith and co-worker ${ }^{40,41}$ and Johnson. ${ }^{42,43}$ The chosen body-fixed frame corresponds to the principal axis system where the $z$-axis is perpendicular to the plane of the molecule, and it is related to the spacefixed frame through the $\alpha, \beta, \gamma$ Eulerian angles. The three internal coordinates, $\rho, \theta$, and $\phi_{\tau}$, are easily related to the Jacobi coordinates $r_{\tau}, R_{\tau}$, and $\Theta_{\tau}$, ${ }^{44}$ where the $\tau$ index identifies the specific atom-diatom arrangement. The method has been previously used to calculate the rovibrational levels of $\mathrm{H}_{3}^{+}$(Refs. 45-47) and it is only briefly outlined below for completeness of presentation.

The expression of the total Hamiltonian in these coordinates can be found elsewhere, ${ }^{39,45}$ so it is not discussed in detail here. The kinetic energy operator consists of the sum $T=T_{\rho}+T_{h}+T_{r}+T_{c}$, where $T_{\rho}$ is a term which depends exclusively on the $\rho$ coordinate, $T_{h}$ describes the motion on the "hypersphere" defined for a given value of $\rho$ by the $\theta$ and $\phi_{\tau}$ coordinates and the two remaining terms $T_{r}+T_{c}$ are those which explicitly depend on the total angular momentum. In particular, these latter terms can be written as

$$
\begin{aligned}
& T_{r}=\frac{\hat{J}_{x}^{2}}{\mu \rho^{2}(1-\sin \theta)}+\frac{J_{y}^{2}}{\mu \rho^{2}(1+\sin \theta)}+\frac{J_{z}^{2}}{2 \mu \rho^{2} \sin ^{2} \theta}, \\
& T_{c}=-\frac{i \hbar}{\mu \rho^{2}} \frac{\cos \theta}{\sin ^{2} \theta} \hat{J}_{z} \frac{\partial}{\partial \phi_{\tau}} .
\end{aligned}
$$

The eigenstates of the total Hamiltonian can be expanded as

$$
\begin{aligned}
\Phi_{k}^{J M} & \left(\rho, \theta, \alpha, \beta, \gamma, \phi_{\tau}\right) \\
& =\frac{4}{\rho^{5 / 2}} \sum_{v m n \Omega} C_{v m n \Omega}^{J M k} W_{\Omega n}^{J M}\left(\alpha, \beta, \gamma, \phi_{\tau}\right) F_{m}^{J \Omega n}(\theta) \varphi_{v}(\rho),
\end{aligned}
$$

where the angular functions $W_{\Omega n}^{J M}$ are of the form

$$
W_{\Omega n}^{J M}\left(\alpha, \beta, \gamma, \phi_{\tau}\right)=\sqrt{\frac{2 J+1}{8 \pi^{2}}} D_{M \Omega}^{J *}(\alpha, \beta, \gamma) \frac{e^{i n \phi_{\tau}}}{\sqrt{2 \pi}},
$$

the $D_{M \Omega}^{J *}$ being Wigner rotation matrices. ${ }^{48}$ In Eq. (3) the $F_{m}^{J \Omega n}(\theta)$ functions satisfy

$$
\left\{\frac{-4}{\sin (2 \theta)} \frac{d}{d \theta} \sin (2 \theta) \frac{d}{d \theta}+\frac{n^{2}+2 n \Omega+\Omega^{2}}{\sin ^{2} \theta}+\frac{2 J(J+1)-2 \Omega^{2}}{\cos ^{2} \theta}-\mathcal{K}(\mathcal{K}+4)\right\} F_{m}^{J \Omega n}(\theta)=0
$$

with $\mathcal{K}=4 m+2 a+2 b, \quad$ where $a=|n+\Omega| / 2 \quad$ and $b=\sqrt{\left[J(J+1)-\Omega^{2}\right] / 2} . F_{m}^{J \Omega n}(\theta)$ are not eigenstates of the full grand angular momentum operator like the hyperspherical harmonics $^{49}$ used in Ref. 37, but are well suited to expand the exact solutions as previously demonstrated for $\mathrm{H}_{3}^{+} \cdot{ }^{45-47}$ The advantage of these functions is that they yield a diagonal representation of the potential matrix elements in the helicity components. 
The $\varphi_{v}(\rho)$ functions appearing in Eq. (3) are the numerical solutions of the one-dimensional differential equation

$$
\left\{-\frac{\hbar^{2}}{2 \mu} \frac{d^{2}}{d \rho^{2}}+V_{r e f}(\rho)-E_{v}\right\} \varphi_{v}(\rho)=0,
$$

where $V_{\text {ref }} \equiv V\left(\rho, \theta=0, \phi_{\tau}=0\right)$ in this case. Equation (6) is solved numerically in a large grid of equispaced values of $\rho$.

The $S_{3}$ permutation group of three identical particles plus the inversion of the spatial coordinates form a group isomorphic with $D_{3 h}$. The HC are particularly well suited for treating the permutation symmetry of triatomic systems with three identical nuclei, a feature which yields a significant reduction in the size of the Hamiltonian matrices for any given IR. For this purpose symmetry adapted functions belonging to each IR of the $D_{3 h}$ group are built as linear combinations of the primitive functions shown in Eq. (4) as explained in Ref. 45.

The good quantum numbers associated with each eigenstate are the total angular momentum, $J$, and the symmetry character $\Gamma$ given by the IR of the $D_{3 h}$ group. Traditionally, several approximate quantum numbers are used for the classification of the eigenstates: ${ }^{50}\left(v_{1}, v_{2}^{\ell}\right), G$ and $U$. Here $v_{1}$ and $v_{2}$ correspond to the symmetric and antisymmetric vibrations while $\ell$ labels the bending. Since in the present treatment the $\varphi_{v}(\rho)$ functions come from a prediagonalization of the symmetric stretch at the equilibrium values of $\theta$ and $\phi_{\tau}, v_{1}$ corresponds to the dominant $v$ in the expansion of Eq. (3). The asymmetric stretch, associated with the $\theta$ coordinate, is analyzed by comparing the full eigenstates with the onedimensional solutions of the problem for $\rho^{e q}, \phi_{\tau}^{e q}$ and $J=0$. The $\ell$ value is associated with the $\phi_{\tau}$ variable and is obtained as

$$
\ell=\left|\Omega_{\max }+n_{\max }\right| / 2,
$$

from the dominant $n$ and $\Omega$ values, $n_{\max }$ and $\Omega_{\max }$ respectively, in the expansion of Eq. (3). Similarly, the $G$ number is also obtained from $n_{\max }$ and $\Omega_{\max }$ as $G=\left|n_{\max }+3 \Omega_{\max }\right| / 2$. Finally, $U$ equals $\ell$ in absolute value and its sign allows to distinguish between the $A_{1}$ and $A_{2}$ components. Since we are using symmetry adapted functions, the latter label is not required to specify the character of the states, and in what follows we shall label the states by $\left(v_{1}, v_{2}^{\ell}\right), J, G$, and $\Gamma$.

In this work we have calculated radial density probability functions by means of the $\mathrm{HC}$ approach. In particular, probability functions in terms of the $\rho$ and $\theta$ coordinates are defined simply as

$$
\mathcal{D}_{k}^{J}(\rho, \theta)=\int d \chi d \phi_{\tau}\left|\Phi_{k}^{J M}\left(\rho, \theta, \alpha, \beta, \gamma, \phi_{\tau}\right)\right|^{2},
$$

being $d \chi=d \alpha d \beta d \gamma$ and $\Phi_{k}^{J M}$ the total wave function from Eq. (3).

Distributions on the $\phi_{\tau}$ and $\theta$ angular coordinates with the same internal quantum numbers but different values of $J$ and $\Omega$ might differ substantially. If we define $\phi_{ \pm}=\left(\gamma \pm \phi_{\tau}\right) / 2$, angular functions from Eq. (4) can be written as

$$
W_{\Omega, n}^{J M}\left(\alpha, \beta, \gamma, \phi_{\tau}\right)=b_{J} e^{i M \alpha} d_{M \Omega}^{J}(\beta) e^{i \Omega_{+} \phi_{+}} e^{i \Omega_{-} \phi_{-}},
$$

where $\Omega_{ \pm}=\Omega \pm n$ and $b_{J}=\sqrt{(2 J+1) /\left(16 \pi^{3}\right)}$. We can thus consider the following reduced wave function to get further insight into the overall structure of the corresponding bound states:

$$
\bar{\Phi}_{k}^{J}\left(\theta, \phi_{+}\right) \sim \sum_{m n \Omega} C_{v_{0} m n \Omega}^{J k} b_{J} e^{i \Omega_{+} \phi_{+}} F_{m}^{J \Omega n}(\theta) \varphi_{v_{0}}\left(\rho_{0}\right),
$$

where we have chosen the projections onto the $v=v_{0}$ basis functions with the largest contributions. The radial $\rho$ coordinate has been fixed at a constant value $\rho_{0}$ and the dependence on the $\alpha, \beta$ and $\phi_{-}$coordinates has been neglected. The real part of this reduced $\bar{\Phi}$ function is defined as the $\Xi_{k}^{J}\left(\theta, \phi_{+}\right)$ function:

$$
\Xi_{k}^{J}\left(\theta, \phi_{+}\right)=\operatorname{Re}\left[\bar{\Phi}_{k}^{J}\left(\theta, \phi_{+}\right)\right],
$$

which can be represented as a function of the $x$ $=\tan (\theta / 2) \cos \phi_{+}$and $y=\tan (\theta / 2) \sin \phi_{+}$coordinates. The wave function is thus expressed in terms of the $\ell$ quantum number via the sum $\Omega+n$. The selection of the component which gives the maximum contribution for the $v$ and $\Omega$ quantum numbers in the expansion of Eq. (3) allows to emphasize the nodal structure of the wave function which might be obscured by the integration of the proper total wave function as defined in Eq. (8).

\section{B. The distributed Gaussian function method}

The method employed here considers the total Hamiltonian as a sum of a purely vibrational term, $J=0$, and the VR part which takes into account the $J>0$ contribution, as $H_{\mathrm{tot}}=H_{\mathrm{vib}}+H_{\mathrm{VR}}$. Both the solution of the rotationless problem and the procedure followed to treat the VR problem with a DGF-based approach have been described in detail elsewhere. ${ }^{12,19,20}$ Thus reviews of previous applications of the method to study the vibrational spectrum of different triatomic species can be found in Refs. 19 and 20. On the other hand, for the VR part, the method we have employed here has been recently discussed in Ref. 12. We therefore refer the reader to those references and shall limit here to the most relevant technical details of the overall method.

Within the DGF approach the total wave function for the rotationless case is expanded in terms of symmetrized basis functions formed with products of Gaussian functions for the interparticle coordinates $\left(R_{1}, R_{2}, R_{3}\right)$. These functions, labeled with the index $k$, are then used to define a symmetry adapted VR basis, $|k ; J \Omega M\rangle,{ }^{7,48,51}$ in which we express the corresponding matrix for $H_{\mathrm{VR}}$ as

$$
\begin{aligned}
& \left\langle k ; J \Omega M\left|H_{\mathrm{VR}}\right| k^{\prime} ; J^{\prime} \Omega^{\prime} M^{\prime}\right\rangle \\
& \quad \approx \sum_{j j^{\prime}} a_{j}^{(k)} a_{j^{\prime}}^{\left(k^{\prime}\right)}\left\langle J \Omega M\left|H_{\mathrm{VR}}^{\dagger}\right| J^{\prime} \Omega^{\prime} M^{\prime}\right\rangle s_{l_{1} l_{1}^{\prime}} s_{l_{2} l_{2}^{\prime}} s_{l_{3} l_{3}^{\prime}},
\end{aligned}
$$

where $a_{j}^{(k)}$ are the coefficients for the rotationless wave function and $H_{\mathrm{VR}}^{\dagger}=H_{\mathrm{VR}}\left(R_{1}^{\dagger}, R_{2}^{\dagger}, R_{3}^{\dagger}\right), R_{i}^{\dagger}$ being the centers of the Gaussian functions obtained as the product of the two DGFs that depend on $R_{i}$. The $s_{l l^{\prime}}$ factors are the overlaps between each pair of Gaussian functions for each radial coordinate used in the basis for the vibrational wave function. 
As explained in detail before, ${ }^{12}$ the procedure consists, in essence, in evaluating all the $R_{1}, R_{2}, R_{3}$-dependent terms of the rotational Hamiltonian (mainly combinations of the $A$, $B$, and $C$ rotational quantities as shown in Eqs. (5) and (6) of Ref. 12) in those intermediate $R_{i}^{\dagger}$ positions. The rotational constants in the $H_{\mathrm{VR}}$ matrix elements, on the other hand, have been calculated according to expressions reported in Ref. 52, obtained within the Eckart conditions ${ }^{53}$ on a reference frame with its $z$ axis along the Jacobi $\mathbf{R}$ vector.

The method employed here is analogous to previous treatments of the VR problem within the context of the perturbation theory in which the total Hamiltonian, $H_{\text {tot }}$, is partitioned in a zero-order contribution containing separately both $H_{\mathrm{vib}}$ and $H_{\mathrm{VR}}$. This Hamiltonian defines the VR representation used to evaluate the different higher order terms in the perturbation series for $H_{\text {tot }}{ }^{2,3,5-7}$ The analogy extends to those approaches based on the Van Vleck transformation ${ }^{54}$ to construct a block diagonal Hamiltonian in the vibrational quantum number. In these methods, the off-diagonal terms account for the interaction between different vibrational manifolds. ${ }^{2,3,48}$ In particular, the DGF scheme proposed here resembles the treatment followed in Ref. 48 to deal with the nonrigid behavior of rotating molecules. Although neither centrifugal distortion nor specific VR coupling terms are explicitly included in the Hamiltonian, the moments of inertia are not strict constants, but quantities which are forced to depend on the vibrational quantum number. ${ }^{2,5}$ The VR interaction within this scheme is therefore introduced by means of the $k \neq k^{\prime}$ terms of the rovibrational Hamiltonian matrix. Diagonalization of such matrix yields the rovibrational spectrum of the complex.

The fluctuations beyond the strict rigid rotor description introduced by the average over the vibrational motion makes the present treatment to bear some resemblance to the VR separation scheme developed by Jellinek and $\mathrm{Li}^{55}$ in their approach, these authors employed the rotation of rigid bodies to describe the instantaneous overall rotation of the nonrigid system.

Although the possibility of the resolution of an exact VR Hamiltonian expressed in terms of internal $R_{1}, R_{2}$, and $R_{3}$ coordinates $^{56}$ certainly exists, our intention here is to test the reliability of the present approximate method to obtain the VR spectra for 3B systems with large values of $J$ without the numerical difficulties of an exact calculation.

The analysis of the symmetry character of the rovibrational spectra is based on the expression of the full symmetry of the wave functions for the rovibrational levels as the product of a vibrational part, $\Gamma_{v}^{\Omega \ell}$, and of a rotational part, $\Gamma_{R}$, as $\Gamma=\Gamma_{v}^{\Omega \ell} \times \Gamma_{R}$. As shown in Ref. 12 the character of $\Gamma_{v}^{\Omega \ell}$ is determined according to the values of the $\ell$ (assigned to the $k$ vibrational states by means of the comparison of the $J=0$ spectra obtained with the HC and DGF approaches) and $\Omega$ quantum numbers. On the other hand, the IR for the rotational part depends on the values of both $\Omega$ and $J$.

\section{POTENTIAL ENERGY SURFACE AND NUMERICAL DETAILS}

The PES employed in the present calculation for the $\mathrm{Ar}_{3}$ cluster has been constructed by means of the HFDID1 semiempirical $\mathrm{Ar}-\mathrm{Ar}$ potential by $\mathrm{Aziz}^{57}$ and the recently reported $a b$ initio potential ${ }^{37}$ for the $3 \mathrm{~B}$ contributions. According to the notation given in Ref. 37, they will be named hereafter as SE2 and AI3, respectively. Extremely time-demanding three variables integrals in the corresponding matrix elements are avoided by a mean value evaluation on the corresponding $R_{i}^{\dagger}$ positions. ${ }^{19,20}$ The validity of such an approximation has been tested for some specific 3B contributions contained in the AI3 potential, by comparison with the corresponding proper integration, yielding almost negligible differences: For the lowest bound states $(k=0-6)$ of the $\mathrm{Ar}_{3}$ trimer, the error does not exceed $5 \times 10^{-3} \%$.

In the DGF calculation, the basis set employed consists of 23 Gaussian functions, where the first one is located at $6.21 a_{0}$, separated by equidistant steps of $0.23 a_{0}$, which generates about 12167 triangular functions. Such basis set differs from the one used in our previous study on the $\mathrm{Ar}_{3}$ trimer $^{32}$ by the specific location of the first Gaussian function and by the total number of DGFs $\left(5.52 a_{0}\right.$ and 73 , respectively). Moreover, in this present case no symmetry considerations are assumed. Since our interest is on a limited number of eigenstates of the rotationless problem, a more reduced basis is sufficient to achieve convergence. The use of this reduced basis set with the same interaction potentials previously used by Baccarelli et al. ${ }^{32}$ yields energy levels which only start to exceed about $0.05 \mathrm{~cm}^{-1}$ for $k=8$ with respect to the results obtained with the larger set.

In the expansion of the total wave function given in Eq. (3) employed in the HC calculation we have considered $v$ $=0, \ldots, 15, k=0, \ldots, 20, n=-36, \ldots, 36$, and all possible $\Omega$ projections. Equation (6) is solved numerically in a grid of 2048 points of equally spaced points in the interval $6<\rho$ $<30 a_{0}$. The corresponding integrals involved in the Hamiltonian matrix are evaluated numerically. A trapezoidal integration is used for $\rho$, while the integral on $\theta$ is carried out by means of a Gauss-Legendre quadrature with a large number of points $(\approx 300)$.

The total number of basis functions used in the $\mathrm{HC}$ calculation is usually quite large for high values of the total angular moment $J$. As an example, for $J=20$ and the totally symmetric representation $\Gamma=A_{1}^{\prime}$, the number of functions is 43 155. Such sizes are too large to be diagonalized using standard variational methods. Instead, we use an iterative procedure based on the Lanczos algorithm ${ }^{58}$ in two steps. The eigenvalues are obtained with a nonorthogonal Lanczos procedure following the method of Cullum and Willoughby. ${ }^{59}$ The eigenstates are then obtained iteratively using the conjugate gradient method ${ }^{60,61}$ in a very efficient way. Typically, between 10000 and 50000 iterations are used to obtain the eigenvalues depending on the size of the Hamiltonian matrices. For the eigenvectors, however, the number of iteration also depends on the density of the spectrum, being of the same order. 
TABLE I. Bound state energies, in $\mathrm{cm}^{-1}$, for the $\mathrm{Ar}_{3}$ cluster at zero angular momentum, $J=0$, obtained by means of the HC and DGF methods with the $\mathrm{SE} 2+\mathrm{AI} 3$ potential. The HC states are shown in the second column labeled with the $\left(v_{1}, v_{2}^{\ell}\right)$ quantum numbers, in the first column. DGF vibrational $k$ states are in the third column. The corresponding IR $\Gamma$ is shown in the fifth column.

\begin{tabular}{|c|c|c|c|c|}
\hline \multicolumn{2}{|c|}{$\mathrm{HC}$} & \multicolumn{3}{|c|}{ DGF } \\
\hline$\left(v_{1}, v_{2}^{\ell}\right)$ & $\begin{array}{c}E \\
\left(\mathrm{~cm}^{-1}\right)\end{array}$ & $k$ & $\begin{array}{c}E \\
\left(\mathrm{~cm}^{-1}\right)\end{array}$ & $\Gamma$ \\
\hline$\left(0,0^{0}\right)$ & -252.23 & 1 & -252.23 & $A_{1}^{\prime}$ \\
\hline$\left(0,1^{1}\right)$ & -229.78 & 2,3 & -229.79 & $E^{\prime}$ \\
\hline$\left(1,0^{0}\right)$ & -221.79 & 4 & -221.79 & $A_{1}^{\prime}$ \\
\hline$\left(0,2^{0}\right)$ & -209.48 & 5 & -209.48 & $A_{1}^{\prime}$ \\
\hline$\left(0,2^{2}\right)$ & -209.32 & 6,7 & -209.33 & $E^{\prime}$ \\
\hline$\left(1,1^{1}\right)$ & -202.58 & 8,9 & -202.58 & $E^{\prime}$ \\
\hline$\left(2,0^{0}\right)$ & -195.97 & 10 & -195.98 & $A_{1}^{\prime}$ \\
\hline$\left(0,3^{1}\right)$ & -193.21 & 11,12 & -193.22 & $E^{\prime}$ \\
\hline$\left(2,1^{0}\right)$ & -191.29 & 13 & -191.28 & $A_{1}^{\prime}$ \\
\hline$\left(0,3^{3}\right)$ & -187.76 & 14 & -187.76 & $A_{2}^{\prime}$ \\
\hline
\end{tabular}

\section{RESULTS}

\section{A. The vibrational spectrum: $J=0$}

We start calculating the bound states of the $\mathrm{Ar}_{3}$ trimer for the rotationless problem, $J=0$, with the $\mathrm{SE} 2+\mathrm{AI} 3$ combination of potential interactions. The energies obtained with the DGF approach for the different $k$ states are compared in Table I with the values, labeled with quantum numbers $\left(v_{1}, v_{2}^{\ell}\right)$, obtained from the HC calculation. Such a comparison was also established in our recent study of Ref. 12, and has been included here to illustrate the correspondence between the $\ell$ quantum number and the DGF $k$ vibrational states. This assignment is crucial to discriminate the symmetry character of the rovibrational states for nonzero values of the total angular momentum. The importance of such an identification relies on the fact that for the $\mathrm{Ar}_{3}$ system, for example, only those rovibrational states from the $A_{1}$ representation are physically acceptable. ${ }^{37}$ By including all bound states in Table I, regardless the precise IR they might belong to, we list the effective seeds for the bound states found in the $J>0$ spectra. In spite of rovibrational states for $J=15$ and 20 shown below do not involve levels with such large $k$ contributing states, we have chosen to extend the rotationless spectrum up to the appearance of the first $A_{2}^{\prime}$ state.

An insight of the radial and angular extension of the $\mathrm{Ar}_{3}$ bound states can be gained with the probability density $\mathcal{D}_{k}^{J=0}(\rho, \theta)$ defined in Eq. (8). In particular, the density functions for the first four states shown in Table I (those labeled with the $\left(0,0^{0}\right),\left(0,1^{1}\right),\left(1,0^{0}\right)$, and $\left(0,2^{0}\right)$ quantum numbers in the $\mathrm{HC}$ calculation and with the $k=1,2-3,4$, and 5 indices in the DGF calculation) obtained with the SE2 + AI3 potential, are shown in the left panels of Fig. 1. In all cases, the $\mathcal{D}_{k}^{J=0}(\rho, \theta)$ functions exhibit a maximum around $\rho \sim 10 \AA$ and spread along the $\theta$ coordinate (up to $\sim 30^{\circ}$ in the case of the $\left(0,2^{2}\right)$ bound state). It is also noticeable the appearance of the first node on the $\rho$ direction for the case of the $\left(1,0^{0}\right)$ state, the first level with excitation in the $v_{1}$ quantum number. Excitations of the two other quantum numbers, $v_{2}$ and $\ell$,

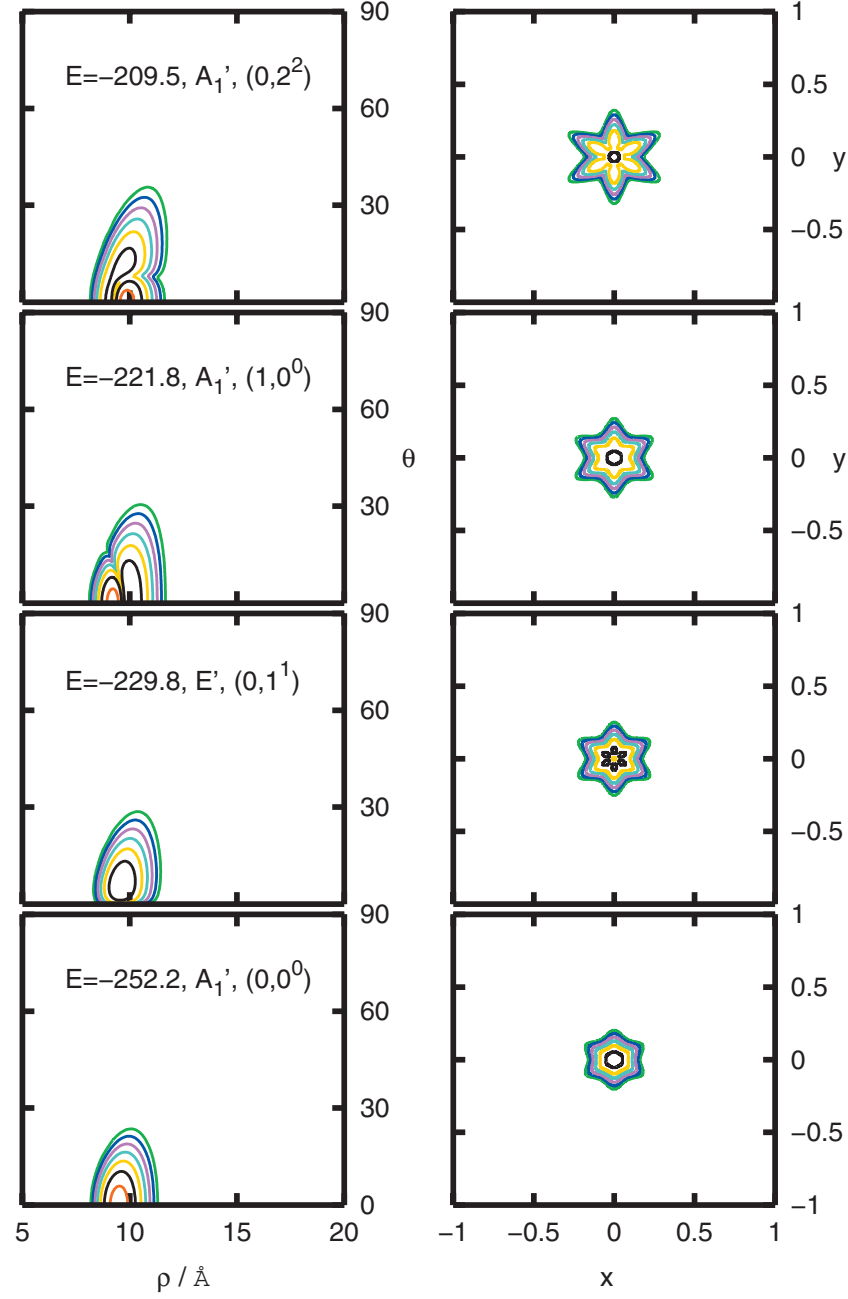

FIG. 1. (Color) Probability density functions $\mathcal{D}_{k}^{J}(\rho, \theta)$ defined in Eq. (8) (left panels) and $\Xi_{k}^{J}$ functions defined in Eq. (11) (right panels) for the first four $\mathrm{Ar}_{3}$ bound states, $\left(0,0^{0}\right),\left(0,1^{1}\right),\left(1,0^{0}\right)$, and $\left(0,2^{2}\right)$, respectively, for $J=0$ from Table I, obtained with the SE2+AI3 potential.

are, however, more difficult to analyze in terms of the $\rho$ and $\theta$ coordinates used in the definition of the $\mathcal{D}_{k}^{J=0}$ probability density functions. Thus, although some distortions with respect to the ground state are appreciable for the $\left(0,2^{2}\right)$ state, a possible node at $\theta \approx 0$ is only suggested for the density function of $\left(0,1^{1}\right)$. Analogously, in the right panels of Fig. 1, we show the $\Xi_{k}^{J=0}$ function defined in Eq. (11) for the same bound states. The structure in the $x$ and $y$ coordinates defined in Sec. II A resembles a star shape in the figure. The inner pattern observed in the close vicinity of the origin, however, seems to display some dependence on the precise value of the $\ell$ quantum number. Thus, the figures for the $\left(0,0^{0}\right)$ and $\left(1,0^{0}\right)$ states, (respectively first and third panels from the bottom in right side of the figure) present some similarities. We will refer to these functions later on when the corresponding density functions for $J>0$ are analyzed.

\section{B. $\mathrm{Ar}_{3}$ bound states for $\mathrm{J}>0$}

In our previous study on the rovibrational spectrum of the $\mathrm{Ar}_{3}$ system $^{12}$ we carried out calculations up to $J=6$, which was the maximum value of the total angular momentum considered in Ref. 37. A further test of the possible 
TABLE II. Comparison between the $\mathrm{Ar}_{3}$ rovibrational levels for $J=15$ corresponding to the $A_{1}^{\prime \prime}$ and $A_{1}^{\prime}$ IRs obtained by means of the present HC and DGF approaches. Values of the $z$-component of $\mathbf{J}$ in the body-fixed frame, $\Omega$, are in the fourth column. Energies, $E_{A B C}$ obtained via the rotational constants calculated in Sec. IV C (see text for details) are included for comparison in the sixth column.

\begin{tabular}{|c|c|c|c|c|c|c|}
\hline \multicolumn{2}{|c|}{$\mathrm{HC}$} & \multicolumn{5}{|c|}{ DGF } \\
\hline$\left(v_{1}, v_{2}^{\ell}\right)$ & $\begin{array}{c}E \\
\left(\mathrm{~cm}^{-1}\right)\end{array}$ & $k$ & $\Omega$ & $\begin{array}{c}E \\
\left(\mathrm{~cm}^{-1}\right)\end{array}$ & $\begin{array}{c}E_{A B C} \\
\left(\mathrm{~cm}^{-1}\right)\end{array}$ & $\Gamma$ \\
\hline$\left(0,0^{0}\right)$ & -244.89 & 1 & 15 & -244.91 & -244.90 & $A_{1}^{\prime \prime}$ \\
\hline$\left(0,0^{0}\right)$ & -242.53 & 1 & 12 & -242.55 & -242.53 & $A_{1}^{\prime}$ \\
\hline$\left(0,0^{0}\right)$ & -240.71 & 1 & 9 & -240.71 & -240.68 & $A_{1}^{\prime \prime}$ \\
\hline$\left(0,0^{0}\right)$ & -239.41 & 1 & 6 & -239.40 & -239.37 & $A_{1}^{\prime}$ \\
\hline$\left(0,0^{0}\right)$ & -238.63 & 1 & 3 & -238.61 & -238.58 & $A_{1}^{\prime \prime}$ \\
\hline$\left(0,1^{1}\right)$ & -222.47 & 2,3 & 14 & -221.81 & -221.80 & $A_{1}^{\prime}$ \\
\hline$\left(0,1^{1}\right)$ & -220.44 & 2,3 & 13 & -221.03 & -221.01 & $A_{1}^{\prime \prime}$ \\
\hline$\left(0,1^{1}\right)$ & -220.06 & 2,3 & 11 & -219.63 & -219.61 & $A_{1}^{\prime \prime}$ \\
\hline$\left(0,1^{1}\right)$ & -218.72 & 2,3 & 10 & -219.02 & -218.99 & $A_{1}^{\prime}$ \\
\hline$\left(0,1^{1}\right)$ & -218.16 & 2,3 & 8 & -217.96 & -217.94 & $A_{1}^{\prime}$ \\
\hline$\left(0,1^{1}\right)$ & -217.50 & 2,3 & 7 & -217.55 & -217.50 & $A_{1}^{\prime \prime}$ \\
\hline$\left(0,1^{1}\right)$ & -216.80 & 2,3 & 5 & -216.86 & -216.80 & $A_{1}^{\prime \prime}$ \\
\hline$\left(0,1^{1}\right)$ & -216.76 & 2,3 & 4 & -216.60 & -216.54 & $A_{1}^{\prime}$ \\
\hline$\left(0,1^{1}\right)$ & -215.98 & 2,3 & 2 & -216.22 & -216.19 & $A_{1}^{\prime}$ \\
\hline$\left(0,1^{1}\right)$ & -215.72 & 2,3 & 1 & -216.11 & -216.10 & $A_{1}^{\prime \prime}$ \\
\hline$\left(1,0^{0}\right)$ & -214.69 & 4 & 15 & -214.71 & -214.70 & $A_{1}^{\prime \prime}$ \\
\hline$\left(1,0^{0}\right)$ & -212.38 & 4 & 12 & -212.40 & -212.38 & $A_{1}^{\prime}$ \\
\hline$\left(1,0^{0}\right)$ & -210.59 & 4 & 9 & -210.60 & -210.57 & $A_{1}^{\prime \prime}$ \\
\hline$\left(1,0^{0}\right)$ & -209.31 & 4 & 6 & -209.32 & -209.28 & $A_{1}^{\prime}$ \\
\hline$\left(1,0^{0}\right)$ & -208.54 & 4 & 3 & -208.55 & -208.50 & $A_{1}^{\prime \prime}$ \\
\hline
\end{tabular}

validity of the approach discussed here is to extend the calculations to even larger values of the total angular momentum. We have thus considered the rovibrational spectrum for $J=15$ and 20 which are clearly much more computationally demanding for an exact calculation. The lowest states $(k$ $\leq 4$ ), for the physically acceptable IRs $A_{1}^{\prime}$ and $A_{1}^{\prime \prime}$, are listed in Tables II and III for $J=15$ and 20, respectively. Our present $\mathrm{HC}$ method has been then extended, with the subsequent increase in the numerical effort, to those larger values of $J$. The agreement between the HC and DGF energy levels is certainly good, the largest observed difference being about $0.6-0.9 \mathrm{~cm}^{-1}$. Despite the noticeable increase in the value of the total angular momentum, these differences are also of the same order of those found for the case of $J=6 .^{12}$

For larger values of $J, \Omega$ is not a good quantum number, and each rovibrational state is, in essence, a mixture of contributions from different values of $\Omega$. In fact, the values included in Tables II and III correspond to those $\Omega$ which give the largest contribution for each $k$ state. This could explain the fact that, as for $J=6,{ }^{12}$ the actual level ordering with decreasing values of the $\Omega$ quantum number for a specific $k$ state in the rovibrational spectrum seems to be altered in some cases. Thus the $k=2,3 ; \Omega=2,1$ levels appear in the HC calculation at lower energies than the $k=2,3 ; \Omega=5,4$ levels.

The analysis of the composition in terms of $k$-states reveals on the contrary that almost negligible mixing is found for the different rovibrational levels existing in the calculated spectra. Apart from those cases belonging to degenerate IRs, for which two vibrational states may be involved, most of the rovibrational states are seen to correspond uniquely to one $k$ state. As a consequence, there is no need to increase enor- mously the $J=0$ basis, including states with $k \gg 0$, in order to ensure the convergence of rovibrational levels associated with a particular rotationless state $k$. In fact, not all the levels shown in Table I are required in order to guarantee the good description of those shown for the $J=15$ and 20 cases.

As we previously did for $J=0$, the $\mathcal{D}_{k}^{J}$ and $\Xi_{k}^{J}$ functions, defined in Eqs. (8) and (11), have been analyzed for the case of nonzero total angular momentum. Among the different levels shown in Table III, probability density functions for the states with energies, $E=-237.4 \mathrm{~cm}^{-1},-218.4 \mathrm{~cm}^{-1}$, and $-207.4 \mathrm{~cm}^{-1}$ have been selected for Fig. 2. These energy levels correspond to specific examples of bound states with the $\left(0,0^{0}\right),\left(0,1^{1}\right)$, and $\left(1,0^{0}\right) \mathrm{HC}$ labels, respectively. It is found that bound states sharing the same $\left(v_{1}, v_{2}^{\ell}\right)$ set of quantum numbers exhibit extremely similar probability densities. Thus, for example, the probability density functions (not shown here) for the first seven bound states listed in Table III are almost identical to those shown in Fig. 2 for the level with energy $E=-237.4 \mathrm{~cm}^{-1}$ (bottom panel). Analogously, the pattern of the $\mathcal{D}_{k}^{J=20}$ and $\Xi_{k}^{J=20}$ functions for the states with a binding energy of $-218.4 \mathrm{~cm}^{-1}$ and $-207.4 \mathrm{~cm}^{-1}$ (in the middle and top panels of Fig. 2, respectively) seems to be repeated for almost the total sequence of states with $\left(0,1^{1}\right)$ and $\left(1,0^{0}\right) \mathrm{HC}$ quantum numbers respectively. Furthermore, the results shown in Figs. 1 and 2 reveal that the high similitude between states defined with the same set of $\left(v_{1}, v_{2}^{\ell}\right)$ quantum numbers also extends when spectra for different values of the total angular momentum are compared. As an example, the correspondence between the probability density functions for the state with $E=-252.2 \mathrm{~cm}^{-1}$ at $J=0$ (see 
TABLE III. Same as Table II for $J=20$.

\begin{tabular}{|c|c|c|c|c|c|c|}
\hline \multicolumn{2}{|c|}{$\mathrm{HC}$} & \multicolumn{5}{|c|}{ DGF } \\
\hline$\left(v_{1}, v_{2}^{\ell}\right)$ & $\begin{array}{c}E \\
\left(\mathrm{~cm}^{-1}\right)\end{array}$ & $k$ & $\Omega$ & $\begin{array}{c}E \\
\left(\mathrm{~cm}^{-1}\right)\end{array}$ & $\begin{array}{c}E_{A B C} \\
\left(\mathrm{~cm}^{-1}\right)\end{array}$ & $\Gamma$ \\
\hline$\left(0,0^{0}\right)$ & -237.36 & 1 & 18 & -237.39 & -237.36 & $A_{1}^{\prime}$ \\
\hline$\left(0,0^{0}\right)$ & -234.50 & 1 & 15 & -234.51 & -234.46 & $A_{1}^{\prime \prime}$ \\
\hline$\left(0,0^{0}\right)$ & -232.17 & 1 & 12 & -232.15 & -232.09 & $A_{1}^{\prime}$ \\
\hline$\left(0,0^{0}\right)$ & -230.36 & 1 & 9 & -230.32 & -230.24 & $A_{1}^{\prime \prime}$ \\
\hline$\left(0,0^{0}\right)$ & -229.08 & 1 & 6 & -229.01 & -228.92 & $A_{1}^{\prime}$ \\
\hline$\left(0,0^{0}\right)$ & -228.31 & 1 & 3 & -228.22 & -228.13 & $A_{1}^{\prime \prime}$ \\
\hline$\left(0,0^{0}\right)$ & -228.05 & 1 & 0 & -227.95 & -227.87 & $A_{1}^{\prime}$ \\
\hline$\left(0,1^{1}\right)$ & -218.43 & 2,3 & 20 & -217.49 & -217.47 & $A_{1}^{\prime}$ \\
\hline$\left(0,1^{1}\right)$ & -215.44 & 2,3 & 19 & -216.36 & -216.33 & $A_{1}^{\prime \prime}$ \\
\hline$\left(0,1^{1}\right)$ & -215.00 & 2,3 & 17 & -214.27 & -214.23 & $A_{1}^{\prime \prime}$ \\
\hline$\left(0,1^{1}\right)$ & -212.73 & 2,3 & 16 & -213.32 & -213.26 & $A_{1}^{\prime}$ \\
\hline$\left(0,1^{1}\right)$ & -212.02 & 2,3 & 14 & -211.58 & -211.51 & $A_{1}^{\prime}$ \\
\hline$\left(0,1^{1}\right)$ & -210.55 & 2,3 & 13 & -210.80 & -210.72 & $A_{1}^{\prime \prime}$ \\
\hline$\left(0,1^{1}\right)$ & -209.59 & 2,3 & 11 & -209.42 & -209.32 & $A_{1}^{\prime \prime}$ \\
\hline$\left(0,1^{1}\right)$ & -208.87 & 2,3 & 10 & -208.81 & -208.71 & $A_{1}^{\prime}$ \\
\hline$\left(0,1^{1}\right)$ & -207.71 & 2,3 & 8 & -207.78 & -207.66 & $A_{1}^{\prime}$ \\
\hline$\left(0,1^{1}\right)$ & -207.68 & 2,3 & 7 & -207.36 & -207.22 & $A_{1}^{\prime \prime}$ \\
\hline$\left(1,0^{0}\right)$ & -207.38 & 4 & 18 & -207.42 & -207.38 & $A_{1}^{\prime}$ \\
\hline$\left(0,1^{1}\right)$ & -206.99 & 2,3 & 2 & -206.52 & -205.90 & $A_{1}^{\prime}$ \\
\hline$\left(0,1^{1}\right)$ & -206.73 & 2,3 & 1 & -206.35 & -205.82 & $A_{1}^{\prime \prime}$ \\
\hline$\left(0,1^{1}\right)$ & -206.36 & 2,3 & 5 & -206.60 & -206.52 & $A_{1}^{\prime \prime}$ \\
\hline$\left(0,1^{1}\right)$ & -205.60 & 2,3 & 4 & -206.35 & -206.25 & $A_{1}^{\prime}$ \\
\hline$\left(1,0^{0}\right)$ & -204.56 & 4 & 15 & -204.60 & -204.54 & $A_{1}^{\prime \prime}$ \\
\hline$\left(1,0^{0}\right)$ & -200.50 & 4 & 9 & -200.51 & -200.41 & $A_{1}^{\prime \prime}$ \\
\hline$\left(1,0^{0}\right)$ & -199.23 & 4 & 6 & -199.24 & -199.11 & $A_{1}^{\prime}$ \\
\hline$\left(1,0^{0}\right)$ & -198.48 & 4 & 3 & -198.46 & -198.34 & $A_{1}^{\prime \prime}$ \\
\hline
\end{tabular}

Fig. 1) and that with $E=-237.4 \mathrm{~cm}^{-1}$ at $J=20$ (see Fig. 2) is clear. Similar links between the states with $E$ $=-229.8 \mathrm{~cm}^{-1}$ and $E=-221.8 \mathrm{~cm}^{-1}$ for $J=0$ and those with $E=-218.4 \mathrm{~cm}^{-1}$ and $E=-207.4 \mathrm{~cm}^{-1}$ respectively are also easy to establish in view of Figs. 1 and 2. As mentioned in Sec. II A, this apparent connection between different states might be obscured unless the $\phi_{+}$and $\phi_{-}$angular coordinates are used.

\section{Rotational constants}

As in previous investigations on molecular trimers such as, for example, $\mathrm{Ar}_{3},{ }^{25,62} \mathrm{Ne}_{2} \mathrm{H}^{-},{ }^{63}$ or $\mathrm{Ne}_{3},{ }^{38}$ we have obtained values for $A, B$, and $C$ associated with each vibrational state $k$. The average values have been calculated by means of the pseudoweights method described in previous occassions. ${ }^{15,19,20}$

The most recently reported values of rotational constants are those given by Karlický et al., obtained from a fit to the expression for the exact bound state energies [see Eq. (16) of Ref. 37]. Among other considerations, such a procedure involves the assumption that the $\mathrm{Ar}_{3}$ molecule can be described as a symmetric top, and therefore the $A$ constant is identical to $B$. Since we have adopted here a more general description in which $A$ and $B$ can differ, a direct comparison with those previously reported $\mathrm{HC}$ rotational constants, requires the average value of these two rotational constants $\bar{B}=(A+B) / 2$. Table IV shows the values for the rotational

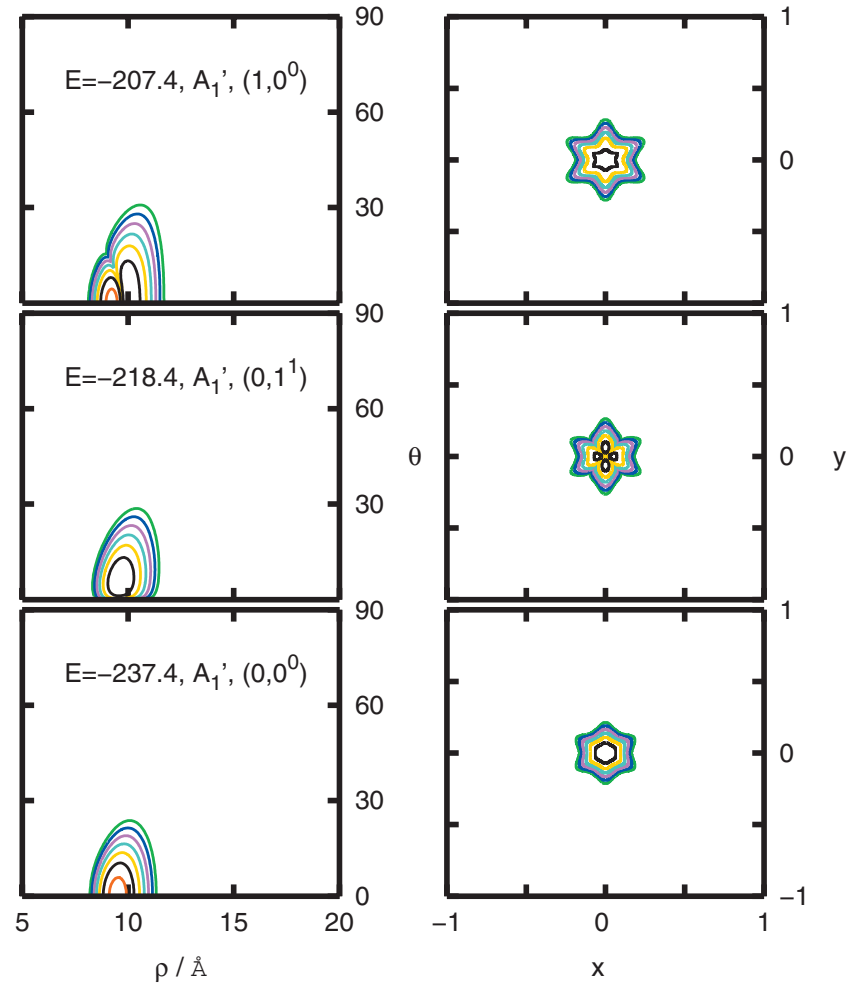

FIG. 2. (Color) Same as Fig. 1 for, from bottom to top, the $\left(0,0^{0}\right)$, $\left(0,1^{1}\right),\left(1,0^{0}\right) \mathrm{Ar}_{3}$ bound states found for $J=20$ with energies $E$ $=-237 \mathrm{~cm}^{-1},-218.4 \mathrm{~cm}^{-1}$, and $-207.4 \mathrm{~cm}^{-1}$, respectively (see Table III). 
TABLE IV. Rotational constants, measured in MHz, obtained with the present DGF approach for the different vibrational $k$ states. The average value $\bar{B}$ between the $A$ and $B$ rotational constants is shown in the fourth column. Values reported, for states denoted with the $v_{1}, v_{2}$, and $l$ quantum numbers in the HC calculation by Karlický et al. are also included for comparison.

\begin{tabular}{|c|c|c|c|c|c|c|c|}
\hline \multicolumn{5}{|c|}{ DGF } & \multicolumn{3}{|c|}{$\mathrm{HC}$} \\
\hline$k$ & A & $B$ & $\bar{B}$ & C & $\left(v_{1}, v_{2}^{\ell}\right)$ & $B$ & $C$ \\
\hline 1 & 1739.26 & 1739.17 & 1739.21 & 861.32 & $\left(0,0^{0}\right)$ & 1738.35 & 863.32 \\
\hline $2-3$ & 1713.43 & 1712.97 & 1713.20 & 837.77 & $\left(0,1^{1}\right)$ & 1697.59 & 785.88 \\
\hline 4 & 1692.88 & 1692.07 & 1692.48 & 831.62 & $\left(1,0^{0}\right)$ & 1691.92 & 834.58 \\
\hline 5 & 1688.91 & 1688.22 & 1688.57 & 812.15 & $\left(0,2^{0}\right)$ & 1596.78 & 809.92 \\
\hline $6-7$ & 1688.16 & 1686.76 & 1687.46 & 809.40 & $\left(0,2^{2}\right)$ & 1694.31 & 627.73 \\
\hline $8-9$ & 1672.23 & 1666.65 & 1669.44 & 806.15 & $\left(1,1^{1}\right)$ & 1630.11 & 644.37 \\
\hline 10 & 1672.66 & 1660.20 & 1666.43 & 782.32 & $\left(2,0^{0}\right)$ & 1653.20 & 782.53 \\
\hline
\end{tabular}

constants obtained in the present DGF method and the HC results from Ref. 37. The differences between the DGF predictions for $C$ and the corresponding $\mathrm{HC}$ values are remarkably small for those states with $\ell=0$ : Less than $4 \mathrm{MHz}$ for the $k=1,4$, and 5 states, and only $0.21 \mathrm{MHz}$ for $k=10$. The corresponding comparison for the $\bar{B}$ values is however slightly more puzzling, since although the agreement is extremely good for the $k=1$ and 4 states, discrepancies between the DGF and HC values are manifestly larger for the other two cases in which $\ell=0: k=5$ and 10 . The rotational constants obtained with the DGF approach for the states with $\ell>0$ differ systematically from the values reported in Ref. 37. Interestingly, these are the cases for which the fitting procedure performed in that study is accompanied with the largest standard deviations. Such deviations were found to increase strongly with $v_{2}$ and $\ell$, which is consistent with the best accord found between the DGF and those $\mathrm{HC}$ rotational constants.

Rotational constants for the ground bound state $\left(0,0^{0}\right)$ have also been calculated in previous studies. By using a pairwise-additive potential, Cooper et $a .^{25}$ obtained $A$ $=1754 \mathrm{MHz}, B=1753$, and $C=872 \mathrm{MHz}$. In a better agreement with the present results, only 2-3 MHz difference, are those reported in Ref. 62, where an AT 3B term was added to the $\mathrm{Ar}-\mathrm{Ar}$ pair interaction: $A=1736.52, B=1736.95$, and $C$ $=859.86 \mathrm{MHz}$. As in those two mentioned examples, ${ }^{25,62}$ the $A$ and $B$ constants for the vibrational ground state are almost identical. In fact the DGF calculation extends the $A \approx B$ similarity up to the $k=6-7$ states. Noticeable differences between both rotational constants are only observed for higher vibrational excitations. This feature is consistent with the major presence of equilateral structures on the overall geometry of the system at its low vibrational states. Given that the present $a b$ initio PES includes $3 \mathrm{~B}$ effects, it has to be concluded that such terms do not imply any substantial deviation from an equilibrium geometry consisting on an equilateral geometry. This is consistent with previous studies on the possible effects of $3 \mathrm{~B}$ terms in the potential function for $\mathrm{Ar}_{3}$, which did not report noticeable differences between the $A$ and $B$ rotational constants. ${ }^{25,62}$

In an attempt to discriminate the possible contributions from the interaction between vibrational states, we have employed the rotational constants for each $k$ state shown in
Table IV to simulate the rovibrational spectra for the cases of $J=15$ and 20 . The calculations were separately performed for each vibrational state neglecting those terms with $k \neq k^{\prime}$ in the rovibrational Hamiltonian matrix. Values of the rotational constants, when needed, were taken from Table IV. The values for the corresponding rovibrational energies, $E_{A B C}$, have been included in Tables II and Table III for comparison with the actual spectra obtained with the DGF method. From the small differences observed between both predicted spectra one can conclude that, at least for the Ar trimer, the vibrational states included in the calculation of the rovibrational energy levels do not significantly interact with each other. Furthermore, the inclusion of such interaction effects yields values slightly more negative than the vibrationally uncoupled energy levels.

\section{DISCUSSION}

The comparison between the $\mathrm{Ar}_{3}$ rovibrational spectra predicted by the DGF method and those obtained with the HC calculations reveals a fairly good agreement between the two methods. Although the approximate and the exact energy values certainly differ more as $J$ increases, the general accord is always remarkable. Thus, the differences between energy levels calculated with both approaches are at most about 0.01 and $0.06 \mathrm{~cm}^{-1}$ for $J=0$ and $J=1$, respectively, and the discrepancies do not exceed 0.6 and $0.9 \mathrm{~cm}^{-1}$ for the two largest values of the total angular momentum investigated in this work, $J=15$ and $J=20$. The issue of the largest value of the total angular momentum for which the DGF-based approach is expected to be still valid is not completely clear. Similar comparisons to those presented here at much larger values of $J$, especially for high energy levels, are limited by the intrinsic numerical difficulty of the exact calculation and the degree of mixing among the corresponding rovibrational levels. A possible alternative way to test the validity of the method could be the investigation of the dependence of different properties of 3B molecular clusters in terms of the temperature. In such studies, thermal averages require the calculation of a large number of partial waves. The comparison with results obtained by means of some other methods as, for example, Monte Carlo simulations, could test the performance of the present approximate approach when a good 
description for high $J$ 's is required. Possible tests have been already envisaged for the present trimer and are currently in progress.

In spite of the satisfactory performance of the DGF approach, it may be of interest, in our opinion, to discuss in more detail some further issues raised by the present study.

Among the different reasons to understand possible discrepancies between the DGF and HC results, one regards the validity of an approach which relies on the use of the eigenstates of the $J=0$ problem as a basis set for the rotational problem. As mentioned in Sec. I, that was the strategy adopted to treat other trimers such as $\mathrm{KCN}^{8}, \mathrm{H}_{2} \mathrm{Ne},{ }^{8} \mathrm{H}_{2} \mathrm{O},{ }^{9}$ $\mathrm{H}_{3}^{+},{ }^{10}$ and $\mathrm{H}_{2} \mathrm{D}^{+} .{ }^{11}$ However, deviations from a rovibrational spectrum obtained under the assumption of a VR separation have been reported for vibrationally excited states of the KCN molecule, where large amplitude vibrations are expected. ${ }^{64}$ In particular, at those highly excited vibrational levels $\mathrm{KCN}$ can explore linear geometries no longer centered at the equilibrium structure, and the system may behave as a hindered rotor, thus precluding any separation between rotation and vibration. That situation, nevertheless, is not likely to happen for the Ar trimer in the energy range we are studying since linear geometries are not expected to play a significant role in the overall structure of the cluster up to $-170 \mathrm{~cm}^{-1} \cdot{ }^{27,32}$ This barrier to linearity is far above the energy levels of the bound states we have considered here, not only for the zero total angular momentum but also for the largest values of $J(J=20)$.

On the other hand, a comparison between the $H_{\mathrm{VR}}$ Hamiltonian employed in the DGF approach and the Hamiltonian written in Eqs. (1) and (2) for the $\mathrm{HC}$ calculation does not yield a straight term-by-term identification. Thus, for example, the results obtained with the $\mathrm{HC}$ method when the $T_{c}$ term [see Eq. (2)] is neglected from the kinetic energy operator, differ significantly from the corresponding rovibrational energy levels predicted by the DGF method shown in Tables II and III. The overall good agreement found with the exact results, however, when both $T_{r}$ and $T_{c}$ terms are considered, indicate that the procedure proposed here constitutes an interesting alternative to those other approximate methods in which centrifugal distortion effects and purely VR terms are explicitly introduced in the total Hamiltonian.

The inspection of the probability density functions obtained in the $\mathrm{HC}$ calculation also reveals interesting features regarding the apparent ability of the DGF approach to describe the $J>0$ spectra of $\mathrm{Ar}_{3}$. In this sense the strong similarities observed between the density functions for the rotationless problem and those for the nonzero total angular momentum cases give support to a treatment in terms of the $J=0$ radial functions. By the definition of the $\Xi_{k}^{J}\left(\theta, \phi_{+}\right)$functions [see Eq. (11)] the above mentioned invariance could be explained as a result of the "conservation" of both the $\Omega$ and $n$ quantum numbers. In other words, it seems as if the $\ell$ number, defined precisely in terms of $\Omega$ and $n$ [see Eq. (7)], turns out to be, at least for $\mathrm{Ar}_{3}$, a quite relevant label to take into account. Thus a possible strategy would be the use of a Hamiltonian with a explicit dependence on such quantum number. Reformulations of the $H_{\text {tot }}$ operator in this direction within the context of the $\mathrm{HC}$ approach have been recently suggested. ${ }^{37}$

The validity of the perturbation approach in the treatment of the VR problem can be affected by the existence of nearly degenerate vibrational states. ${ }^{3}$ The mixing of different vibrational states used to describe the wave function of a VR state was analyzed in detail, for example, in the study of the water molecule performed by Chen $e t a l .{ }^{9}$ The effects due to the coupling between different vibrational states are also taken into account within the present DGF method since the VR energy spectrum is finally obtained as a result of the diagonalization of the total Hamiltonian in the rovibrational basis function $|k ; J \Omega M\rangle$. In this sense, the present study suggests that the effective vibration-vibration coupling seems to be negligible for the Ar trimer, at least for the energy range covered here. As mentioned in Sec. IV, the $\mathrm{Ar}_{3}$ VR spectra for the different values of $J$ considered in this study appear to be formed by separate subsets of bound states associated with specific $k$ states with nearly zero interaction among each other. The analysis of the corresponding wave functions reveals the almost complete absence of interaction among the $J=0$ vibrational states. It would be then interesting to apply the present scheme to molecules exhibiting nearby vibrational states. This scenario could be approached by artificial modifications of the present PES in order to induce some congestion in the purely vibrational spectrum. Attempts in this direction are presently in progress. The convergence of the method may also be affected by this aspect, since under such conditions the number of vibrational states required to guarantee the correct description of the rovibrational states correlated with a particular $k$-state could certainly increase.

Rotational constants have been calculated by means of different techniques in previous studies of the Ar trimer. Thus, for example, the $B$ and $C$ constants for the different bound states can be obtained from analytical expressions for the energy levels of the system assumed as symmetric top. $^{37,65}$ Alternatively, the rotational constants can be estimated by averaging the moments of inertia for each vibrational state over the full wave function. ${ }^{23,25,62}$ Interestingly, discrepancies with the rotational constants obtained when $\mathrm{Ar}_{3}$ is treated as a rigid body in its equilibrium geometry were interpreted as a measure of the degree of floppiness of the molecule. ${ }^{65}$ A similar analysis ${ }^{23}$ of the possible nonrigidity of the Ar trimer reported mean deviation values from the average moments of inertia between $7 \%$ and $15 \%$ even for the lowest bound states. Analogously the absence of noticeable differences between the actual rovibrational levels and the predicted spectra, $E_{A B C}$, obtained here for $J=15$ and 20 with the values of the rotational constants for each vibrational at $J=0$ provides further support to a description in terms of a rigid rotor assumption.

An important issue on the calculation of the rotational constants $^{52,62}$ regards the use of the Eckart conditions ${ }^{53}$ for the reference frame chosen to describe the rotating system. Previous estimations of the rotational constants for $\mathrm{Ar}_{3}$ (Ref. 25) were found to be affected by significant errors for T-shaped trimers ${ }^{62}$ due to the fact that the chosen axis system had one inertial axis fixed along the intermolecular vector. ${ }^{66}$ 
Improved formulas for the expectation values of the rotational constants were then obtained by applying the Eckart conditions. ${ }^{52}$ The values for $A, B$, and $C$ have been obtained here with a reference frame defined within the Eckart conditions. Results obtained with the DGF approach without verifying such requirements yield considerable differences between the $A$ and $B$ constants.

\section{CONCLUSIONS}

The case of the rotating Ar trimer has been studied by means of an approximate variational quantum method based on a separate treatment of the purely vibrational (or rotationless) and rotational cases. The solution of the vibrational problem is carried out by means of a procedure which employs the $R_{1}, R_{2}$, and $R_{3}$ internal coordinates and describes the interparticle distances by distributed Gaussian functions. The eigenstates of the vibrational Hamiltonian are then used in combination with standard rotational basis functions to describe the rotational Hamiltonian. The interaction between the different vibrational states is included in this scheme by means of the off-diagonal blocks of the corresponding Hamiltonian matrix. The radial dependence existing in the rotational Hamiltonian is averaged over the different bound states of the vibrational spectrum, therefore allowing for certain deviations from a simpler description in terms of a rigid rotor.

The comparison with results obtained using hyperspherical coordinate calculations reveals that the predicted spectra for a wide range of values for the total angular momentum $(J \leq 20)$ are remarkably well reproduced. Although the differences with the exact energy levels is found to increase, the accord is quite good: lower than $0.9 \mathrm{~cm}^{-1}$ for some bound states of the most demanding situation analyzed here, $J=20$.

The rotational constants for each vibrational state have been calculated. The small differences between the $A$ and $B$ constants for the lower bound states of the $J=0$ case reveal that the overall geometry is close to an equilateral triangle. The agreement with recently reported results using the same potential energy surface is quite good, especially with those states for which some assumptions as the condition of symmetric top for the $\mathrm{Ar}_{3}$ system seem to be satisfied. A comparison of the actual spectra with those rovibrational levels obtained with the rotational constants $A, B$, and $C$ averaged for each vibrational state suggests minimal interaction among the eigenstates of the $J=0$ case.

The analysis of different probability densities for large values of the total angular momentum shows evidence of a strong influence of the geometry found in the $J=0$ spectrum. This could give support to the validity of the present approximate approach to treat rotating trimers, such as the $\mathrm{Ar}_{3}$ system. Further applications to other three-body systems would help to establish the limits of the proposed method. In this sense, trimers with a more congested vibrational spectrum for which the effects of nearby vibrational states can be important constitute an interesting challenge for this method.

\section{ACKNOWLEDGMENTS}

Support from the Spanish MEC and CSIC under Grant Nos. FIS2007-62006 and 200650I134 was acknowledged. We would like to sincerely acknowledge Professor J. M. Hutson for useful discussions and providing us with a copy of Ref. 65. The support from Italy-Spain Collaborative Action is also acknowledged.

\footnotetext{
${ }^{1}$ See, for example, early reviews of G. D. Carney, L. L. Sprandel, and C. W. Kern, Adv. Chem. Phys. 37, 305 (1978); S. Carter and N. C. Handy, Comput. Phys. Rep. 5, 115 (1986); J. Tennyson, ibid. 4, 1 (1986); Z. Bacić and J. C. Light, Annu. Rev. Phys. Chem. 40, 469 (1989).

${ }^{2}$ E. B. Wilson and J. B. Horward, J. Chem. Phys. 4, 260 (1936).

${ }^{3}$ E. B. Wilson, J. Chem. Phys. 4, 313 (1936).

${ }^{4}$ E. B. Wilson, J. Chem. Phys. 5, 617 (1937).

${ }^{5}$ W. H. Shaffer and H. H. Nielsen, Phys. Rev. 56, 188 (1939).

${ }^{6}$ H. H. Nielsen, Rev. Mod. Phys. 23, 90 (1951).

${ }^{7}$ H. W. Kroto, Molecular Rotation Spectra (Dover, New York, 1992).

${ }^{8}$ J. Tennyson and B. T. Sutcliffe, J. Chem. Phys. 77, 4061 (1982).

${ }^{9}$ C.-L. Chen, B. Maessen, and M. Wolfsberg, J. Chem. Phys. 83, 1795 (1985).

${ }^{10}$ V. Spirko, P. Jensen, P. R. Bunker, and A. Cejchan, J. Mol. Spectrosc.
} 112, 183 (1985).

${ }^{11}$ J. Tennyson and B. T. Sutcliffe, Mol. Phys. 58, 1067 (1986).

${ }^{12}$ M. Márquez-Mijares, T. González-Lezana, O. Roncero, S. Miret-Artés, G. Delgado-Barrio, and P. Villarreal, Chem. Phys. Lett. 460, 417 (2008).

${ }^{13}$ I. P. Hamilton and J. C. Light, J. Chem. Phys. 84, 306 (1986).

${ }^{14}$ T. González-Lezana, J. Rubayo-Soneira, S. Miret-Artés, F. A. Gianturco, G. Delgado-Barrio, and P. Villarreal, Phys. Rev. Lett. 82, 1648 (1999).

${ }^{15}$ T. González-Lezana, J. Rubayo-Soneira, S. Miret-Artés, F. A. Gianturco, G. Delgado-Barrio, and P. Villarreal, J. Chem. Phys. 110, 9000 (1999).

${ }^{16}$ S. Orlandini, I. Baccarelli, and F. A. Gianturco, Comput. Phys. Commun. 180, 384 (2009).

${ }^{17}$ I. Baccarelli, G. Delgado-Barrio, F. A. Gianturco, T. González-Lezana, S. Miret-Artés, and P. Villarreal, Europhys. Lett. 50, 567 (2000).

${ }^{18}$ I. Baccarelli, G. Delgado-Barrio, F. A. Gianturco, T. González-Lezana, S. Miret-Artés, and P. Villarreal, Phys. Chem. Chem. Phys. 2, 4067 (2000).

${ }^{19}$ T. González-Lezana, S. Miret-Artés, G. Delgado-Barrio, P. Villarreal, J. Rubayo-Soneira, I. Baccarelli, F. Paesani, and F. A. Gianturco, Comput. Phys. Commun. 145, 156 (2002).

${ }^{20}$ I. Baccarelli, F. A. Gianturco, T. González-Lezana, G. Delgado-Barrio, S. Miret-Artés, and P. Villarreal, Phys. Rep. 452, 1 (2007)

${ }^{21}$ T. González-Lezana, D. López, S. Miret-Artés, F. A. Gianturco, G. Delgado-Barrio, and P. Villarreal, Chem. Phys. Lett. 335, 105 (2001).

${ }^{22}$ D. M. Leitner, R. S. Berry, and R. M. Whitnell, J. Chem. Phys. 91, 3470 (1989).

${ }^{23}$ T. R. Horn, R. B. Gerber, J. J. Valentini, and M. A. Ratner, J. Chem. Phys. 94, 6728 (1991).

${ }^{24}$ D. M. Leitner, J. D. Doll, and R. M. Whitnell, J. Chem. Phys. 94, 6644 (1991).

${ }^{25}$ A. R. Cooper, S. Jain, and J. M. Hutson, J. Chem. Phys. 98, 2160 (1993).

${ }^{26}$ C. Chakravarty, R. J. Hinde, D. M. Leitner, and D. J. Wales, Phys. Rev. E 56, 363 (1997)

${ }^{27}$ N. J. Wright and J. M. Hutson, J. Chem. Phys. 110, 902 (1999).

${ }^{28}$ D. Blume and C. H. Greene, J. Chem. Phys. 113, 4242 (2000).

${ }^{29}$ D. Blume, C. H. Greene, and B. D. Esry, J. Chem. Phys. 113, 2145 (2000).

${ }^{30}$ S. Garashchuk and J. C. Light, J. Chem. Phys. 114, 3929 (2001).

${ }^{31}$ P.-N. Roy, J. Chem. Phys. 119, 5437 (2003).

${ }^{32}$ I. Baccarelli, F. A. Gianturco, T. González-Lezana, G. Delgado-Barrio, S. Miret-Artés, and P. Villarreal, J. Chem. Phys. 122, 144319 (2005).

${ }^{33}$ G. Chalasiński, M. Szczęśniak, and R. A. Kendall, J. Chem. Phys. 101, 8860 (1994)

${ }^{34}$ R. Bukowski and K. Szalewicz, J. Chem. Phys. 114, 9518 (2001).

${ }^{35}$ A. Dullweber and D. J. Wales, Mol. Phys. 94, 651 (1998).

${ }^{36}$ V. F. Lotrich and K. Szalewicz, J. Chem. Phys. 106, 9688 (1997).

${ }^{37}$ F. Karlický, B. Lepetit, R. Kalus, and F. X. Gadea, J. Chem. Phys. 126, 174305 (2007).

${ }^{38}$ M. Salci, S. B. Levin, N. Elander, and E. Yarevsky, J. Chem. Phys. 129, 134304 (2008).

${ }^{39}$ R. T. Pack and G. A. Parker, J. Chem. Phys. 90, 3511 (1989).

${ }^{40}$ F. T. Smith, J. Math. Phys. 3, 735 (1962). 
${ }^{41}$ R. C. Whitten and F. T. Smith, J. Math. Phys. 9, 1103 (1968).

${ }^{42}$ B. R. Johnson, J. Chem. Phys. 73, 5051 (1980).

${ }^{43}$ B. R. Johnson, J. Chem. Phys. 79, 1916 (1983).

${ }^{44}$ R. T. Pack and G. A. Parker, J. Chem. Phys. 87, 3888 (1987).

${ }^{45}$ A. Aguado, O. Roncero, C. Tablero, C. Sanz, and M. Paniagua, J. Chem. Phys. 112, 1240 (2000).

${ }^{46}$ C. Sanz, O. Roncero, C. Tablero, A. Aguado, and M. Paniagua, J. Chem. Phys. 114, 2182 (2001).

${ }^{47}$ E. Cuervo-Reyes, J. Rubayo-Soneira, A. Aguado, M. Paniagua, C. Tablero, C. Sanz, and O. Roncero, Phys. Chem. Chem. Phys. 4, 6012 (2002).

${ }^{48}$ R. N. Zare, Angular Momentum (Wiley, New York, 1988).

${ }^{49}$ L. Wolniewicz, J. Chem. Phys. 90, 371 (1989).

${ }^{50}$ J. K. G. Watson, J. Mol. Spectrosc. 103, 350 (1984).

${ }^{51}$ C. H. Townes and A. L. Schawlow, Microwave Spectroscopy (Dover, New York, 1975).

${ }^{52}$ A. Ernesti and J. M. Hutson, Chem. Phys. Lett. 222, 257 (1994).

${ }^{53}$ C. Eckart, Phys. Rev. 47, 552 (1935).
${ }^{54}$ J. H. Van Vleck, Phys. Rev. 33, 467 (1929).

${ }^{55}$ J. Jellinek and D. H. Li, Phys. Rev. Lett. 62, 241 (1989).

${ }^{56}$ V. H. Diehl, S. Flügge, U. Schröder, A. Völkel, and A. Weiguny, Z. Phys. 162, 1 (1961).

${ }^{57}$ R. A. Aziz, J. Chem. Phys. 99, 4518 (1993).

${ }^{58}$ C. Lanczos, J. Res. Natl. Bur. Stand. 45, 255 (1950).

${ }^{59}$ J. K. Cullum and R. A. Willoughby, Lanczos Algorithms for Large Symmetric Eigenvalues Computations (Birkhäuser, Boston, 1985).

${ }^{60}$ C.-E. Fröberg, in Numerical Mathematics: Theory and Computer Applications (The Benjamin/Cummings, New York, 1985).

${ }^{61}$ R. E. Wyatt, Adv. Chem. Phys. 73, 231 (1989).

${ }^{62}$ A. Ernesti and J. M. Hutson, J. Chem. Phys. 103, 3386 (1995).

${ }^{63}$ I. Baccarelli, F. A. Gianturco, T. González-Lezana, G. Delgado-Barrio, S. Miret-Artés, and P. Villarreal, J. Phys. Chem. A 110, 5487 (2006).

${ }^{64}$ J. Tennyson and A. van der Avoird, J. Chem. Phys. 76, 5710 (1982).

${ }^{65}$ N. J. Wright, Ph.D. thesis, University of Durham, 1998, Chap. 7.

${ }^{66}$ J. M. Hutson, J. A. Beswick, and N. Halberstadt, J. Chem. Phys. 90, 1337 (1989). 